\title{
Cutaneous Odontogenic Sinus: An Inflammatory Mimicker of Squamous Cell Carcinoma and Epidermal Cysts
}

\author{
Atieh Jibbe, MD; Stanislav N. Tolkachjov, MD
}

\begin{abstract}
An odontogenic cutaneous sinus tract (OCST) of dental origin is an uncommon and frequently misdiagnosed lesion that is caused by chronic periodontitis. Given that OCSTs often lack symptoms, are located on the lower face, and can have notable variations in clinical appearance, they can be mistaken for more common dermatologic diagnoses such as squamous cell carcinomas (SCCs) and cysts. We present 2 patients with OCSTs who were referred for cutaneous surgery for a rendered diagnosis of an SCC and epidermal cyst. A proper diagnosis was rendered after a high index of suspicion, and clinicopathologic correlation led to additional testing and eventual referral to oral surgery for an OCST.
\end{abstract}

Cutis. 2020;106:196-198.

\section{Clinical Challenge}

An odontogenic cutaneous sinus tract (OCST) of dental origin is an uncommon occurrence and is most commonly caused by chronic periodontitis, specifically a periapical abscess due to chronic dental infection. ${ }^{1,2}$ Odontogenic cutaneous sinus tract commonly is misdiagnosed due to a lack of symptoms on presentation, location, and variations in clinical appearance mimicking other lesions. Patients with OCSTs typically present with a fixed, erythematous, crusty, and nontender nodule with periodic drainage that easily can be mistaken for a pustule, furuncle, cyst, foreign-body lesion, squamous cell carcinoma (SCC), basal cell carcinoma, or granulomatous disorder. ${ }^{3}$ It becomes challenging for dermatologists to correctly diagnose these lesions and refer for proper evaluation and treatment.

\section{Practice Gap}

It is estimated that half of patients with an extraoral fistula are treated with multiple dermatologic surgical operations, radiotherapy, antibiotic therapy, and chemotherapy before the correct diagnosis is made. ${ }^{1}$ Thus, proper identification of these lesions is crucial for prognosis and treatment. The most common locations for OCSTs are the mandibular, submandibular, and cervical skin. ${ }^{1,2}$ Given these locations, patients with OCSTs commonly present to the dermatology office for evaluation. Education regarding the clinical presentation, histopathology, and proper evaluation and further referral for treatment is essential for dermatologists.

\section{Tools and Technique for Diagnosis}

We present 2 patients with OCSTs who were referred for cutaneous surgery for an SCC and epidermal cyst, but the proper diagnosis was rendered after an index of suspicion and clinicopathologic correlation led to additional testing and eventual referral for imaging.

Patient 1-A 68-year-old woman presented for Mohs micrographic surgery (MMS) of a biopsy-proven SCC on the chin. The tumor cleared after 2 MMS stages (Figure 1A). Due to notable inflammation in each stage, the slides were sent to a pathologist who confirmed clear margins. Within 2 weeks of MMS, the wound began to dehisce (Figure 1B). The patient presented 4 months later with a crusted ulcerated nodule at the MMS site (Figure 1C). A biopsy showed likely recurrence of SCC. Upon presentation to the Mohs surgeon, the nodule felt fixed to the underlying jaw, and the patient was noted to have poor dentition. The patient was sent for computed tomography (CT), which showed focal thinning of the mandible, likely postsurgical, and clear maxillary sinuses. Due to the clinical appearance and anatomic location of the lesion, a request was made for a second read of the $\mathrm{CT}$, specifically looking for an OCST at the prior surgical site. With this information, the radiologist noted an OCST extending from the mandible to the lesion, reported as a periapical lucency (representing a periapical abscess) at a

From Epiphany Dermatology, Dallas, Texas.

The authors report no conflict of interest.

Correspondence: Stanislav N. Tolkachjov, MD, 9191 Kyser Way, Ste B, Frisco, TX 75033 (stan.tolkachjov@gmail.com).

doi:10.12788/cutis.0081 
mandibular tooth with a dental sinus draining into the soft tissues. The patient was started on antibiotics and referred to an oral surgeon for OCST excision.

Patient 2-A 62-year-old man presented with an inflamed subcutaneous nodule on the left anterior neck. A biopsy showed a ruptured cyst, and the patient was referred for excision. Clinical examination revealed a subcutaneous nodule fixed to the lower portion of the mandible (Figure 2A) that exhibited a rubbery retraction when pulled (Figure 2B). After a discussion about the atypical feel and appearance of this cyst, the patient preferred to undergo excision. During excision, the lesion felt deep and fixed with retraction (Figure 2C). With intraoperative re-evaluation of the clinical scenario and location, the patient was sent for CT. The initial read noted clear maxillary and ethmoid sinuses, with no mention of an OCST. After discussing the clinical history and suspicion specifically for an OCST with the radiologist, the re-read showed notable inflammation and decay of the tooth adjacent to the area of interest. An OCST was diagnosed, and the patient was sent to an oral surgeon for excision after antibiotics were prescribed.

\section{Practice Implications}

Odontogenic cutaneous sinus tracts commonly are misdiagnosed due to variations in clinical presentations resembling more common cutaneous diagnoses, nonspecific histopathologic findings, and lack of dental symptoms or concerns about dentition. Clinically, an OCST presents as a fixed, red, crusty, nontender nodule with intermittent draining. With palpation of the involved area, the clinician may feel a cord of tissue connecting the skin lesion intraorally. ${ }^{2,4}$ A clinician should have a high index of suspicion for an OCST when evaluating fixed lesions of the lower face, jawline, and neck due to the possibility of a dental origin, ${ }^{1}$ which is important because an OCST can have similar clinical findings to lesions such as congenital fistulas, pustules, cysts, osteomyelitis, foreignbody granulomas, pyogenic granulomas, syphilis, metastatic carcinomas, basal cell carcinomas, and SCCs. ${ }^{2,4} \mathrm{~A}$ PubMed search of articles indexed for MEDLINE using the terms Mohs, MMS, chemosurgery, odontogenic sinus, odontogenic cutaneous sinus tract, and dental sinus yielded only 2 OCSTs that were referred for MMS in the last 30 years, both of which were in the nasolabial fold/medial
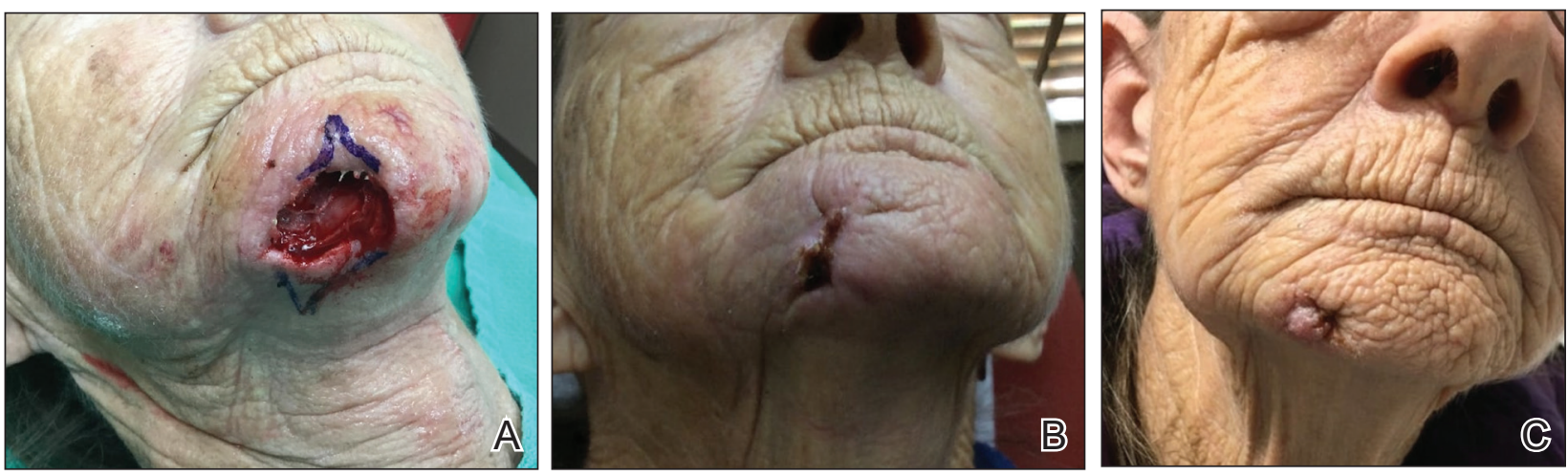

FIGURE 1. A, Defect after initial Mohs micrographic surgery (MMS) for a crusted ulcerated nodule on the mental chin mimicking a recurrent squamous cell carcinoma. B, Wound dehiscence 2 weeks after MMS. C, Clinical appearance of a fixed subcutaneous nodule concerning for recurrence at the same lateral chin location 4 months after MMS, consistent with an odontogenic cutaneous sinus tract.

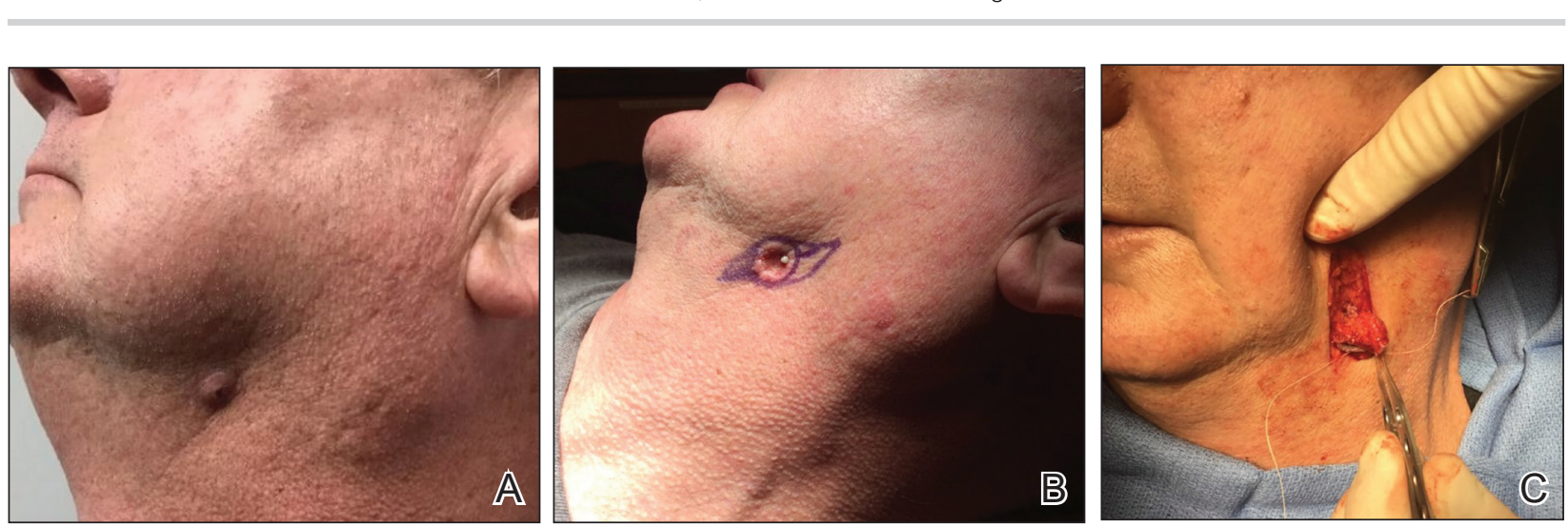

FIGURE 2. A, Initial lesion of the lateral jawline/superior neck clinically concerning for a squamous cell carcinoma that was diagnosed as a ruptured cyst on initial histopathology. B, Appearance after a biopsy at presentation showed an ulcerated subcutaneous nodule fixed to the lower portion of the mandible that exhibited a rubbery retraction upon pulling, consistent with an odontogenic cutaneous sinus tract (OCST) of the anterior neck. C, Intraoperative view showed a deep tract fixed to the underlying jaw, consistent with an OCST of the anterior neck. 
malar cheek. ${ }^{2,4}$ Histopathologic findings of an OCST are nonspecific; a mixed or granulomatous inflammatory infiltrate, granulation tissue, and scarring can be seen. ${ }^{1}$ Pseudocarcinomatous/pseudoepitheliomatous hyperplasia of the epidermis can be seen and cause histologic misinterpretation for an SCC. ${ }^{2}$ Given that these findings are nonspecific without a clinical context, even with a histopathologic diagnosis of SCC or cyst, a clinical suspicion for an OCST should lead to an intraoral examination. Imaging can be ordered to look for an OCST in the area of interest. Although panoramic or periapical radiography with or without dental probes/radiopaque markers commonly have been used, more recent literature has suggested that CT may be superior to radiographs for making an OCST diagnosis. ${ }^{1,3}$ If imaging is not consistent with the clinically suspected OCST, we recommend directly contacting the radiologist to explain the clinical history and even refresh his/her suspicion for this diagnosis.

If a diagnosis of an OCST is made, oral antibiotics can be prescribed, though the use of antibiotics has been controversial. For severe odontogenic infections, typically beta-lactam antibiotics, cephalosporins, metronidazole, clindamycin, moxifloxacin, or erythromycin can be given for 7 days or until 3 days after symptoms have resolved. ${ }^{5}$ Although antibiotics can bring temporary resolution, it is imperative to treat the source of infection to prevent recurrence. It is crucial for these patients to be referred to an oral surgeon for evaluation and treatment of OCST by either a root canal or tooth extraction.

\section{Final Thoughts}

We present this pearl on the diagnosis and management of an OCST, also known as a dental sinus, to better assist clinicians in making this diagnosis. With an index of suspicion as well as intraoral and radiologic evaluations, a proper diagnosis may be rendered, potentially avoiding unnecessary cutaneous surgery. In addition, we highlight the importance of communication between the clinician and the radiologist to directly look for OCST in the area of concern and consider a re-read of the images when clinical suspicion does not correlate with the radiology report.

\section{REFERENCES}

1. Bai J, Ji AP, Huang MW. Submental cutaneous sinus tract of mandibular second molar origin. Int Endod J. 2014;47:1185-1191.

2. Cohen PR, EliezriYD. Cutaneous odontogenic sinus simulating a basal cell carcinoma: case report and literature review. Plast Reconstr Surg. 1990:86:123-127.

3. Gregoire C. How are odontogenic infections best managed? J Can Dent Assoc. 2010;76:a37.

4. Bodner L, Bar-Ziv J. Cutaneous sinus tract of dental originimaging with a dental CT software programme. Br J Oral Maxillofac Surg. 1998;36:311-313.

5. Peermohamed S, Barber D, Kurwa H. Diagnostic challenges of cutaneous draining sinus tracts of odontogenic origin: a case report. Dermatol Surg. 2011;37:1525-1527. 